\title{
Effect of Heat Generation and Radiation on Heat Transfer in a Micropolar Fluid over a Stretching Sheet with Newtonian Heating
}

\author{
S. Vijaya Lakshmi ${ }^{1}$, T. Amarnatha Reddy ${ }^{2}$, M. Suryanarayana Reddy ${ }^{3}$ \\ ${ }^{1}$ Lecturer in Mathemetics, NTR Govt. Degree College, Vayalapadu, AP, India \\ ${ }^{2}$ Dept. of Physics, Aditya College of Engineering, Madanapalle, AP, India \\ ${ }^{3}$ Dept. of Mathematics, JNTUA College of Engineering, Pulivendala , AP, India \\ *Corresponding Author: S. Vijaya Lakshmi, Lecturer in Mathemetics, NTR Govt. Degree College, \\ Vavalapadu. AP. India

\begin{abstract}
The steady, two dimensional flow of incompressible micropolar fluid over a stretching sheet with Newtonian heating in the presence of heat generation and radiation. Using the similarity transformations, the governing equations have been transformed into a system of ordinary differential equations. These differential equations are highly nonlinear which cannot be solved analytically. Therefore, fifth-order RungeKutta integration scheme has been used for solving it. Numerical results are obtained for the skin-friction coefficient, the couple wall stress and the local Nusselt number as well as the velocity, microrotation and temperature profiles for different values of the governing parameters, namely, material parameter, heat generation parameter and radiation parameter.
\end{abstract}

Keywords: Heat Transfer, micropolar fluid, Stretching surface, heat generation, radiation.

\section{INTRODUCTION}

Understanding the flow of non-Newtonian fluids is a problem of great interest of researchers and practical importance. There are several natural and industrial applications of such fluids, for instance volcanic lava, molten polymers, drilling mud, oils, certain paints, poly crystal melts, fluid suspensions, cosmetic and food products and many others. The flow dynamics of non-Newtonian fluids can be described by non-linear relationships between the shear stress and shear rate. Further these fluids have sheared dependent viscosity. In literature there exist many mathematical models with different constitutive equations involving different set of empirical parameters. The micropolar fluid model is adequate for exocitic lubricants, animal blood, liquid crystals with rigid molecules, certain biological fluids and colloidal or suspensions solutions. The micromotion of fluid elements, spin inertia and the effects of the couple stresses are very important in micropolar fluids $[1,2]$. The fluid motion of the micropolar fluid is characterized by the concentration laws of mass, momentum and constitutive relationships describing the effect of couple stress, spin-inertia and micromotion. Hence the flow equation of micropolar fluid involves a micro -rotation vector in addition to classical velocity vector. In micropolar fluids, rigid particles in a small volume element can rotate about the centred of the volume element. The micropolar fluids in fact can predict behaviour at microscale and rotation is independently explained by a microrotation vector. More interesting aspects of the theory and application of micropolar fluids can be found in the books of Eringen [3] and Lukazewicz [4] and in some studies of Peddieson and McNitt [5] Willson [6], Siddheshwar and Manjunath [7].

In Newtonian heating, the rate of heat transfer from the bouncing surface with a finite heating capacity is proportional to the local temperature surface which is usually termed as conjugate convective flow (see Merkin [8], Lesnic et al. [9], Chaudhary and Jain [10] and Salleh et al. [11] numerically investigated the boundary layer flow of viscous fluid over a stretched surface in the regime of Newtonian heating. Numerical solution of the differential system is obtained by Keller box method. Recently, Qasim [12] studied the heat Transfer in a micropolar fluid over a stretching sheet with newtonian heating. Uddin et al. [13] studied the MHD free convective boundary layer flow of a nanofluid past a flat vertical plate with newtonian heating. 
Effect of Heat Generation and Radiation on Heat Transfer in a Micropolar Fluid over a Stretching Sheet with Newtonian Heating

The heat source/sink effects in thermal convection, are significant where there may exist a high temperature differences between the surface (e.g. space craft body) and the ambient fluid. Heat generation is also important in the context of exothermic or endothermic chemical reactions. Singh et al. [14], investigated the effect of volumetric heat generation/absorption on mixed convection stagnation point flow on an isothermal vertical plate in porous media. Das and his co-workers [15] analyzed the effect of mass transfer on MHD flow and heat transfer past a vertical porous plate through a porous medium under oscillatory suction and heat source. Ibrahim et al.[16] have examined the effects of unsteady MHD micropolar fluids over a vertical porous plate through a porous medium in the presence of thermal and mass diffusion with a constant heat source. Rehman and sattar [17] have analyzed the effect of magnetohydrodynamic convective flow of a micropolar fluid past a continuously moving porous plate in the presence of heat generation/ absorption.

The study of radiation effects on the various types of flows is quite complicated. In the recent years, many authors have studied radiation effects on the boundary layer of radiating fluids past a plate. Raptis [18] studied the flow of a micropolar fluid past a continually moving plate by the presence of radiation. The radiation effect on heat transfer of a micropolar fluid past unmoving horizontal plate through a porous medium was studied by Abo-Eldahab and Ghonaim [19]. Ishak [20] investigated that the thermal boundary layer flow over a stretching sheet in a micropolar fluid with radiation effect. Uddin el [21] investigated the MHD forced convective laminar boundary layer flow from a convectively heated moving vertical plate with radiation and transpiration effect. Recently, Mohammed Ibrahim and Bhaskar Reddy [22] studied the mass transfer and thermal radiation effects over a stretching sheet in a micropolar fluid with heat generation.

The present study investigates the steady, two dimensional flow of an incompressible micropolar fluid over a stretching sheet with Newtonian heating in the presence of heat generation, radiation. Using the similarity transformations, the governing equations have been transformed into a set of ordinary differential equations, which are nonlinear and cannot be solved analytically, therefore, fifth order Runge-Kutta integration scheme has been used for solving it. The results for velocity, microrotation and temperature functions are carried out for the wide range of important parameters namely, material parameter, heat generation parameter and radiation parameter. The skin friction, the couple wall stress and the rate of heat transfer have also been computed.

\section{MATHEMATiCAl Formulation}

A steady boundary layer flow of an incompressible micropolar fluid induced by a stretching surface is considered. The sheet is stretched with a velocity $u_{w}(x)=c x$ (where $c$ is a real number). It is assumed that the Dufour effects are neglected in the energy equation. It is further assumed that the fluid properties are taken to be constant except for the density variation with the temperature in the buoyancy term. Under the usual boundary layer approximation, the governing equations are

Continuity equation

$$
\frac{\partial u}{\partial x}+\frac{\partial v}{\partial y}=0
$$

Linear momentum equation $u \frac{\partial u}{\partial x}+v \frac{\partial u}{\partial y}=\left(v+\frac{\kappa}{\rho}\right) \frac{\partial^{2} u}{\partial y^{2}}+\frac{\kappa}{\rho} \frac{\partial N}{\partial y}$

Angular momentum equation $\rho j\left(u \frac{\partial N}{\partial x}+v \frac{\partial N}{\partial y}\right)=\gamma * \frac{\partial^{2} N}{\partial y^{2}}-\kappa\left(2 N+\frac{\partial u}{\partial y}\right)$

Energy equation

$$
u \frac{\partial T}{\partial x}+v \frac{\partial T}{\partial y}=\frac{k}{\rho c_{p}} \frac{\partial^{2} T}{\partial y^{2}}-\frac{1}{\rho c_{p}} \frac{\partial q_{r}}{\partial y}+\frac{Q_{0}}{\rho c_{p}}\left(T-T_{\infty}\right)
$$

The boundary conditions for the velocity, temperature and concentration fields are $u=u_{w}(x)=c x, v=0, N=-n \frac{\partial u}{\partial y}, \frac{\partial T}{\partial y}=h_{s} T$ at $\quad y=0 \quad u \rightarrow 0, N \rightarrow 0, T \rightarrow T_{\infty} \quad$ as $\quad y \rightarrow \infty$ 
Effect of Heat Generation and Radiation on Heat Transfer in a Micropolar Fluid over a Stretching Sheet with Newtonian Heating

where $u$ and $v$ are the velocity components parallel to the $x$ and $y$ axes, respectively, $\rho$ the fluid density, $v$ the kinematic viscosity, $T$ is temperature, $N$ the microrotation or angular velocity, $T_{\infty}$ is the ambient temperature, $c_{p}$ the specific heat, $k$ the thermal conductivity of the fluid, $Q_{0}$ is the heat generation or absorption rate constant, $j=(v / c)$ is microinertia per unit mass, $\gamma^{*}=(\mu+\kappa / 2) j$ and $\kappa$ are the spin gradient viscosity and vortex viscosity, respectively. Here $\kappa=0$ corresponds to situation of viscous fluid and the boundary parameter $n$ varies in the range $0 \leq n \leq 1$. Here $n=0$ corresponds to the situation when microelements at the stretching sheet are unable to rotate and denotes weak concentrations of the microelements at sheet. The case $n=1 / 2$ corresponds to the vanishing of anti-symmetric part of the stress tensor and it shows weak concentration of microelements and the case $n=1$ is for turbulent boundary layer flows, $h_{s}$ is the heat transfer coefficient.

By using the Rosseland approximation the radiative heat flux $q_{r}$ is given by

$$
q_{r}=-\frac{4 \sigma_{1}}{3 k *} \frac{\partial T^{4}}{\partial y}
$$

Where $\sigma_{1}$ is the Stefan -Boltzmann constant and $k^{*}$ is the mean absorption coefficient. It should be noted that by using the Rosseland approximation, the present analysis is limited to optically thick fluids. If temperature differences within the flow are significantly small, then equation (2.4) can be linearised by expanding $T^{4}$ into the Taylor series about $T_{\infty}$, which after neglect higher order terms takes the form:

$$
T^{4} \cong 4 T_{\infty}^{3} T-3 T_{\infty}^{4}
$$

In view of equations (2.8) and (2.9), eqn. (2.6) reduces to

$u \frac{\partial T}{\partial x}+v \frac{\partial T}{\partial y}=\left(\frac{k}{\rho c_{p}}+\frac{16 \sigma_{1} T_{\infty}^{3}}{3 k^{*} \rho c_{p}}\right) \frac{\partial^{2} T}{\partial y^{2}}+\frac{Q_{0}}{\rho c_{p}}\left(T-T_{\infty}\right)$

The continuity equation (2.1) is satisfied by the Cauchy Riemann equations

$u=\frac{\partial \psi}{\partial y}$ and $v=-\frac{\partial \psi}{\partial x}$

where $\psi(x, y)$ is the stream function.

In order to transform equations (2.2), (2.3) (2.8) and (2.5) into a set of ordinary differential equations, the following similarity transformations and dimensionless variables are introduced.

$\eta=\sqrt{\frac{c}{v}} y, N=\sqrt{\frac{c}{v}} \operatorname{cxg}(\eta), u=c x f^{\prime}(\eta), v=-\sqrt{c v} f(\eta)$

$\theta(\eta)=\frac{T-T_{\infty}}{T_{\infty}}, K=\frac{\kappa}{\mu}, \operatorname{Pr}=\frac{\mu c_{p}}{\rho} \beta=\frac{Q_{0}}{\rho c_{p} c}, R=\frac{16 \sigma_{1} T_{\infty}^{3}}{3 k * k \rho c_{p}}$

where $f(\eta)$ is the dimensionless stream function, $\theta$ is the dimensionless temperature, $N$ is the dimensionless microrotation, $\eta$ is the similarity variable, $K$ is the material parameter, $\beta$ is the heat generation parameter, $R$ is the radiation parameter and $\operatorname{Pr}$ is the Prandtl number. In view of equations (2.9) and (2.10), the equations (2.2), (2.3), (2.8) and (2.5) transform into $(1+K) f^{\prime \prime \prime}+f f^{\prime \prime}-\left(f^{\prime}\right)^{2}+K g^{\prime}=0$

$(1+K / 2) g^{\prime \prime}+f g^{\prime}-f^{\prime} g-K\left(2 g+f^{\prime \prime}\right)=0$

$(1+R) \frac{1}{\operatorname{Pr}} \theta^{\prime \prime}+f \theta^{\prime}+\beta \theta=0$ 
Effect of Heat Generation and Radiation on Heat Transfer in a Micropolar Fluid over a Stretching Sheet with Newtonian Heating

The corresponding boundary conditions are $f(0)=0, f^{\prime}(0)=1, g(0)=-n f^{\prime \prime}(0), \theta^{\prime}(0)=-\gamma[1+\theta(0)] f^{\prime}=g=\theta=0$ as $\eta \rightarrow \infty$

where the primes denote differentiation with respect to $\eta$ and $\gamma=h_{s} \sqrt{v / c}$ his the conjugate parameter for Newtonian heating.

The physical quantities of interest are the skin friction coefficient $C_{f x}$, the local couple wall stress $M_{w x}$ and the local Nusselt number $N u_{x}$ which are defined as

$$
\begin{aligned}
& C_{f x}=\frac{1}{\rho u_{w}^{2}}\left[(\mu+\kappa)\left(\frac{\partial u}{\partial y}\right)_{y=0}+\kappa(N)_{y=0}\right] \\
& =(1+(1-n) K) \operatorname{Re}_{x}^{-1 / 2} f^{\prime \prime}(0) \\
& M_{w x}=\gamma^{*}\left(\frac{\partial N}{\partial y}\right)_{y=0}=\frac{\gamma^{*} u_{w}}{v} g^{\prime}(0)
\end{aligned}
$$

$$
N u_{x}=-\frac{x}{T_{w}-T_{\infty}}\left(\frac{\partial T}{\partial y}\right)_{y=0}=\gamma\left[1+\frac{1}{\theta(0)}\right] \operatorname{Re}_{x}^{2}
$$

Where $\operatorname{Re}_{x}=\left(c x^{2} / v\right)$ is the local Reynolds number.

Our main aim is to investigate how the values of $C_{f x}$ and $N u_{x}$ vary in terms of the various parameters.

\section{Solution of the Problem}

The set of non-linear coupled differential equations (2.11)-(2.13) subject to the boundary conditions equation (2.14) constitute a two-point boundary value problem. In order to solve these equations numerically we follow most efficient numerical shooting technique with fifth-order Runge-Kuttaintegration scheme. In this method it is most important to choose the appropriate finite values of $\eta \rightarrow \infty$. To select $\eta_{\infty}$ we begin with some initial guess value and solve the problem with some particular set of parameters to obtain $f$ " and $1 / \theta(0)$. The solution process is repeated with another large value of $\eta_{\infty}$ until two successive values of $f^{\prime \prime}$ and $1 / \theta(0)$ differ only after desired digit signifying the limit of the boundary along $\eta$. The last value of $\eta_{\infty}$ is chosen as appropriate value of the limit $\eta \rightarrow \infty$ for that particular set of parameters. The three ordinary differential equations (2.11)(2.13) were first formulated as a set of seven first-order simultaneous equations of seven unknowns following the method of superposition [23]. Thus, we set

$$
\begin{aligned}
& y_{1}=f, y_{2}=f^{\prime}, y_{3}=f^{\prime \prime}, y_{4}=g, y_{5}=g^{\prime}, y_{6}=\theta, y_{7}=\theta^{\prime} \\
& y_{1}{ }^{\prime}=y_{2}, y_{2}{ }^{\prime}=y_{3} \\
& y_{1}(0)=0, y_{2}(0)=1 \\
& y_{3}{ }^{\prime}=\frac{1}{1+K}\left(y_{2}{ }^{2}-y_{1} y_{3}-K y_{5}\right) \\
& y_{3}(0)=\delta_{1} \\
& y_{4}{ }^{\prime}=y_{5}, y_{4}(0)=-n y_{3}(0) \\
& y_{5}{ }^{\prime}=\frac{1}{1+K / 2}\left[y_{2} y_{4}-y_{1} y_{5}+K\left(2 y_{4}+y_{3}\right)\right] \\
& y_{5}(0)=\delta_{2} \\
& y_{6}{ }^{\prime}=y_{7}, y_{6}(0)=-\gamma\left(1+y_{6}(0)\right) \\
& y_{7}{ }^{\prime}=-\frac{\operatorname{Pr}}{1+R}\left[y_{1} y_{7}+\beta y_{6}\right] \\
& y_{7}(0)=\delta_{3}
\end{aligned}
$$


Effect of Heat Generation and Radiation on Heat Transfer in a Micropolar Fluid over a Stretching Sheet with Newtonian Heating

Eqs. (2.11) - (2.13) then reduced into a system of ordinary differential equations, i.e., where $\delta_{1}, \delta_{2}$ and $\delta_{3}$ are determined such that it satisfies $y_{2}(\infty) \rightarrow 0, y_{4}(\infty) \rightarrow 0$ and $y_{6}(\infty) \rightarrow 0$. The shooting method is used to guess $\delta_{1}, \delta_{2}$ and $\delta_{3}$ until the boundary conditions $y_{2}(\infty) \rightarrow 0, y_{4}(\infty) \rightarrow 0$ and $y_{6}(\infty) \rightarrow 0$ are satisfied. Then the resulting differential equations can be integrated by fifth-order Runge-Kutta integration scheme. The above procedure is repeated until we get the results up to the desired degree of accuracy, $10^{-6}$.

\section{RESULTS AND DISCUSSION}

The governing equations (2.11) - (2.13) subject to the boundary conditions (2.14) are integrated as described in section 3. In order to get a clear insight of the physical problem, the velocity, temperature and concentration have been discussed by assigning numerical values to the parameters encountered in the problem. The effects of various parameters on velocity profiles in the boundary layer are depicted in Figs. 1-2. The effects of various parameters on Angular velocity profiles in the boundary layer are depicted in Figs.3-4. The effects of various parameters on temperature profiles in the boundary layer are depicted in Figs. 5-10.

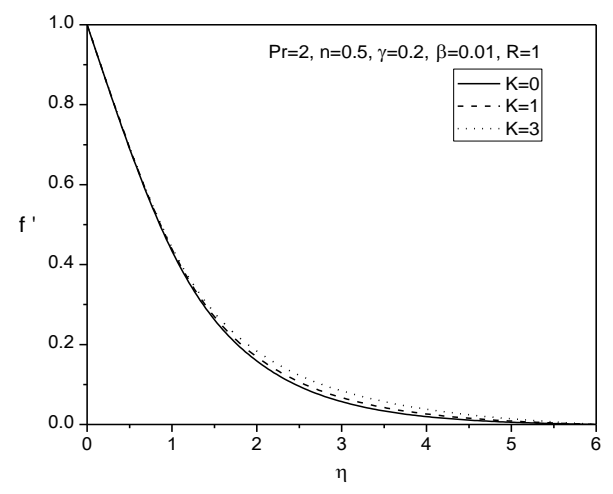

Fig.1 Velocity for different values of $K$

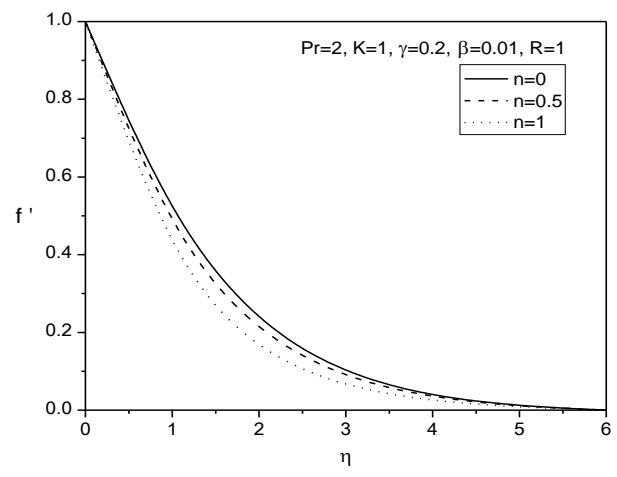

Fig.2 Velocity for different values of $n$

The effect of material parameter $(K)$ on the velocity is illustrated in Fig.1. It is noticed that the velocity increases with increasing values of the material parameter. Fig. 2 shows the variation of the velocity with the boundary parameter $(n)$. It is noticed that the velocity thickness decreases with an increase in the boundary parameter.

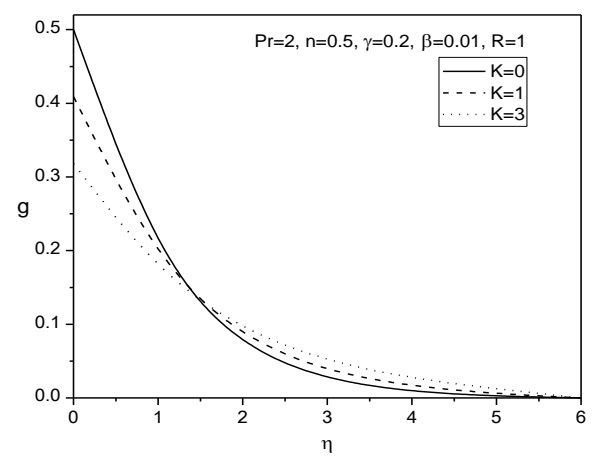

Fig.3 Angular velocity for different values of $K$

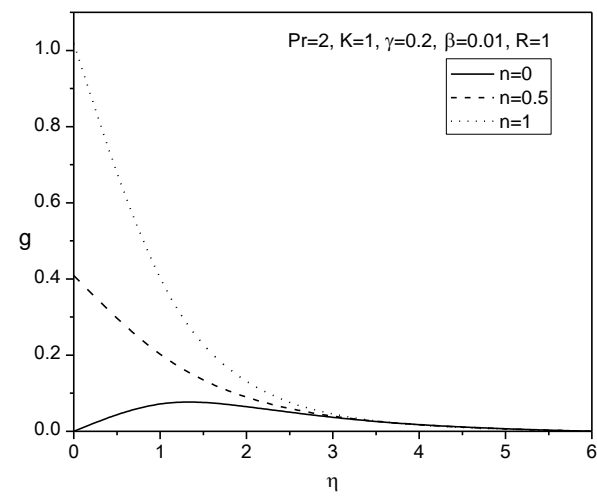

Fig.4 Angular velocity for different values of $n$

Fig. 3 depicts the angular velocity with the material parameter. It is noticed that the angular velocity thickness decreases neat the wall and increases for away the wall with an increase in the material parameter. The effect of the boundary parameter on the angular velocity is illustrated in Fig.4. It is observed that as the boundary parameter increases, the angular velocity boundary layer thickness increases. 
Effect of Heat Generation and Radiation on Heat Transfer in a Micropolar Fluid over a Stretching Sheet with Newtonian Heating

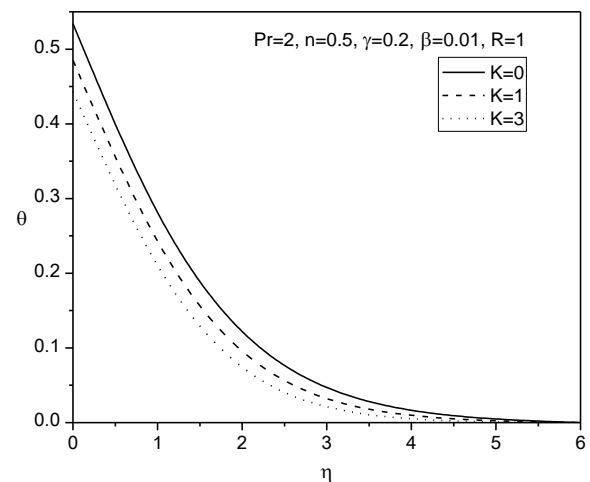

Fig.5 Temperature for different values of $K$

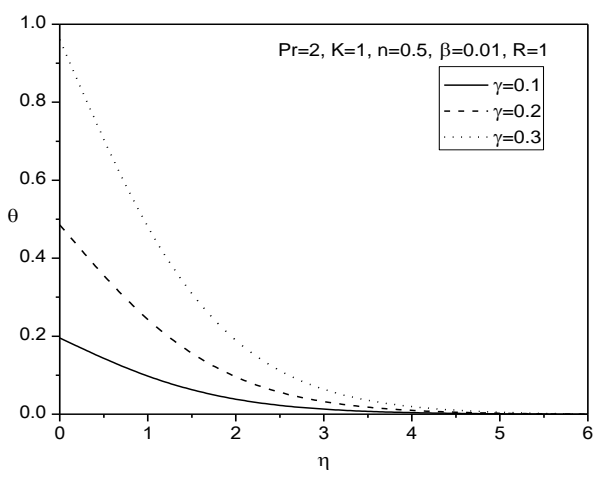

Fig.7 Temperature for different values of $\gamma$

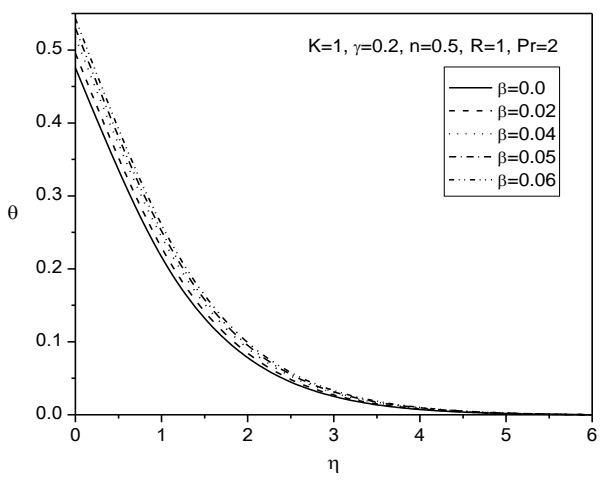

Fig.9 Temperature for different values of $\beta$

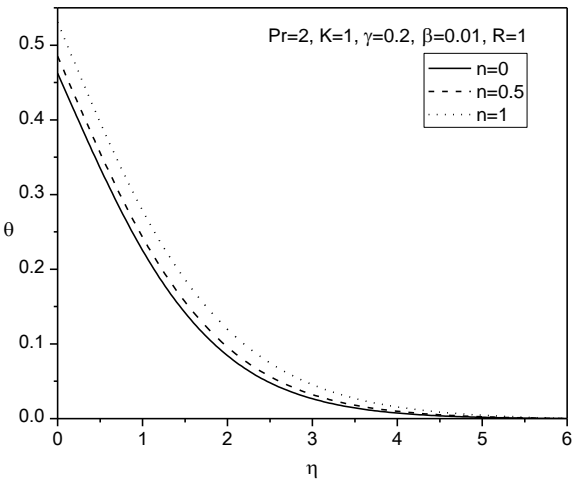

Fig.6 Temperature for different values of $n$

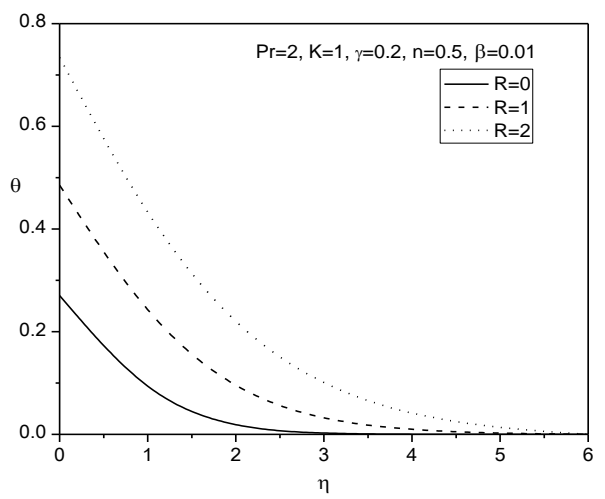

Fig.8 Temperature for different values of $R$

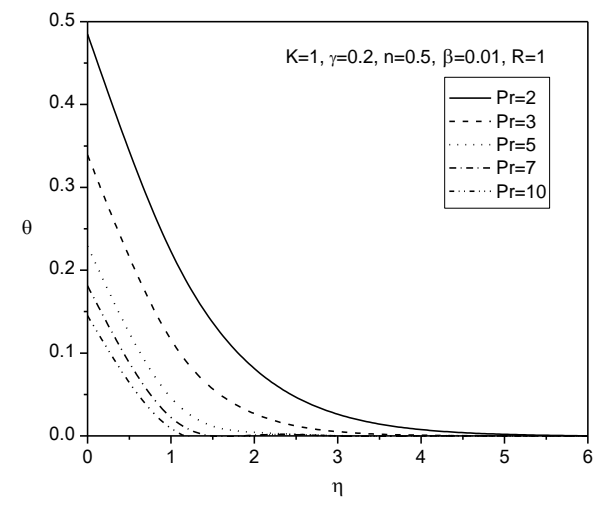

Fig.10 Temperature for different values of $\mathrm{Pr}$

Fig. 5 depicts the thermal boundary-layer with the material parameter. It is noticed that the thermal boundary layer thickness decreases with an increase in the material parameter. Fig.6 illustrates the effect of the boundary parameter on the temperature. It is noticed that as the boundary parameter increases, the temperature increases. Fig. 7 depicts the thermal boundary-layer with the conjugate parameter $(\gamma)$. It is noticed that the thermal boundary layer thickness increases with an increase in the conjugate parameter. Fig. 8 depicts the thermal boundary-layer with the radiation parameter $(R)$. It is noticed that the thermal boundary layer thickness increases with an increase in the radiation parameter. Fig. 9 shows the variation of the thermal boundary-layer with the heat generation parameter $(\beta)$. It is observed that the thermal boundary layer thickness increases with an increase in the heat generation parameter. Fig. 10 shows the variation of the thermal boundary-layer with the Prandtl number $(\mathrm{Pr})$. It is noticed that the thermal boundary layer thickness decreases with an increase in the Prandtl number. 
Effect of Heat Generation and Radiation on Heat Transfer in a Micropolar Fluid over a Stretching Sheet with Newtonian Heating

Table 1. Comparison of $\operatorname{Re}_{x}^{1 / 2} C_{f x}$ for different values of $K$ when $\operatorname{Pr}=0.7, Q=R=0, \gamma=1$.

\begin{tabular}{|l|l|l|l|l|}
\hline \multirow{3}{*}{$K$} & \multicolumn{3}{|l|}{$\mathrm{Re}_{x}^{1 / 2} C_{f x}$} \\
\cline { 2 - 5 } & Qasim et al. [12] & \multicolumn{2}{l|}{ Present study } \\
\cline { 2 - 5 } & $n=0$ & $n=0.5$ & $n=0$ & $n=0.5$ \\
\hline 0 & -1.000000 & -1.000000 & -1.00006 & -1.00006 \\
1 & -1.367872 & -1.224741 & -1.36832 & -1.22514 \\
2 & -1.621225 & -1.414218 & -1.62249 & -1.41972 \\
4 & -2.004133 & -1.732052 & -2.02322 & -1.74890 \\
\hline
\end{tabular}

For validation of the numerical method used in this study, results for skin friction were compared with those of Qasim et al. [12] for various values of $K$. The quantitative comparison is shown in Table 1 and it is found to be in excellent agreement. It is found that the magnitude of skin friction coefficient increases for large values of $K$.

Table 2. Computation of $\operatorname{Re}_{x}^{-1 / 2} N u_{x}$ for different values of $K, \operatorname{Pr}, R$, $\beta$ when $\gamma=1$.

\begin{tabular}{|l|l|l|l|l|}
\hline$K$ & $P r$ & $R$ & $\beta$ & $\operatorname{Re}_{x}^{-1 / 2} N u_{x}$ \\
\hline 0 & 0.7 & 1 & 0.01 & 0.304278 \\
1 & 0.7 & 1 & 0.01 & 0.323934 \\
2 & 0.7 & 1 & 0.01 & 0.337452 \\
4 & 0.7 & 1 & 0.01 & 0.355226 \\
\hline 1 & 0.7 & 1 & 0.01 & 0.323934 \\
1 & 1 & 1 & 0.01 & 0.395543 \\
1 & 2 & 1 & 0.01 & 0.612275 \\
1 & 3 & 1 & 0.01 & 0.789814 \\
\hline 1 & 0.7 & 0 & 0.01 & 0.487271 \\
1 & 0.7 & 0.5 & 0.01 & 0.379749 \\
1 & 0.7 & 1 & 0.01 & 0.323934 \\
1 & 0.7 & 2 & 0.01 & 0.268446 \\
\hline 1 & 0.7 & 1 & 0.0 & 0.329214 \\
1 & 0.7 & 1 & 0.2 & 0.208165 \\
1 & 0.7 & 1 & 0.4 & 0.0331674 \\
1 & 0.7 & 1 & 0.6 & -0.284422 \\
\hline
\end{tabular}

Table 2 shows the variation of the Nusselt number with for different values of material parameter, Prandtl number, radiation parameter and heat generation parameter. It is observed that the Nusselt number increases with an increase in the material parameter or Prandtl number and decreases with increasing the radiation parameter or heat generation parameter.

\section{Conclusions}

In the present prater, steady, two dimensional flow of an incompressible micropolar fluid over a stretching sheet with Newtonian heating by taking heat generation and radiation effects into account, are analyzed. The governing equations are approximated to a system of non-linear ordinary differential equations by similarity transformation. Numerical calculations are carried out for various values of the dimensionless parameters of the problem. It has been found that

1. The velocity and the angular velocity increases as well as, the temperature decreases with an increase in the material parameter.

2. The heat generation and radiation enhances the temperature.

3. The skin friction enhances the material parameter.

4. The heat generation and radiation reduces the heat transfer rate.

\section{REFERENCES}

[1] Eringen, A.C., (1964), Simple micropolar fluids, Int J Eng Sci., Vol.2, pp.205-207.

[2] Eringen, A.C., (1966), Theory of micropolar fluids, J Math Mech., Vol.16, pp.1-18.

[3] Eringen, A.C., (2001), Microcontinuum field theories II, Fluent Media. Springer, Newyork. 
Effect of Heat Generation and Radiation on Heat Transfer in a Micropolar Fluid over a Stretching Sheet with Newtonian Heating

[4] Lukaszewicz, G., (1999), Micropolar fluids: Theory and applications, Brikhauser Basel.

[5] Peddieson, J., McNitt, R.P., (1970), Boundary layer theory for micropolar fluid, Recent Adv Eng Sci., Vol.5, pp.405-426.

[6] Willson, A.J., (1970), Boundary layers in micropolar liquids, Proc. Camb Phil Soc., Vol.67, pp.469-476.

[7] Siddheshwar, P.G., Manjunath, S., (2000), Unsteady convective diffusion with heterogeneous chemical reaction in a plane-Poiseuille flow of a micropolar fluid, Int J Eng Sci., Vol.38, pp.765-783.

[8] Merkin, J.H., (1999), Natural-convection boundary-layer flow on a vertical surface with Newtonian heating, Int. J. Heat. Fluid Flow, Vol.15, pp.392-398.

[9] Lesnic, D., Ingham, D.B., Pop, I., (1999), Free convection boundary layer flow along a vertical surface in a porous medium with Newtonian heating, Int. J. Heat Mass Transf., Vol.42, pp.2621-2627.

[10] Chaudhary, R.C., Jain, P., (2007), An exact solution to the unsteady free convection boundary layer flow past an impulsive started vertical surface with Newtonian heating, J. Eng. Phys., Vol.80, pp.954-960.

[11] Salleh, M.Z., Nazar, R., Pop, I., (2010), Boundary layer flow and heat transfer over a stretching sheet with Newtonian heating, J Taiwan Inst Chem Eng., Vol.41, pp.651-655.

[12] Qasim, M., Khan, I., Shafie, S., (2013), Heat Transfer in a Micropolar Fluid over a Stretching Sheet with Newtonian Heating, PLoS ONE, Vol.8(4): e59393. doi:10.1371/journal.pone.0059393, pp.1-6.

[13] Uddin, M.J., Khan, W.A., Ismail, A.I., (2012), MHD Free Convective Boundary Layer Flow of a Nanofluid past a Flat Vertical Plate with Newtonian Heating Boundary Condition, PLoS ONE, Vol.7(11): e49499. doi:10.1371/journal.pone.0049499, pp.1-8.

[14] Uddin, M.J., Khan, W.A., Ismail, A.I.M., (2013), MHD Forced Convective Laminar Boundary Layer Flow from a Convectively Heated Moving Vertical Plate with Radiation and Transpiration Effect, PLoS ONE, Vol.8(5): e62664. doi:10.1371/journal.pone.0062664,pp.1-10.

[15] Singh, G., Sharma, P.R., and Chamkha, A.J., (2010), Effect of Volumetric Heat Generation/Absorption on Mixed Convection Stagnation Point Flow on an Isothermal Vertical Plate in Porous Media, Int. J. Industrial Mathematics, Vol.2(2), pp.59-71.

[16] Das, S.S., Satapathy, A., Das, J.K., and Panda, J.P., (2009), Mass transfer effects on MHD flow and heat transfer past a vertical porous plate through a porous medium under oscillatory suction and heat source, Int. J. Heat Mass Transfer, Vol.52, pp.5962-5969.

[17] Ibrahim, F.S., Hassanien, I.A., Bakr, A.A., (2004), unsteady magnetohydrodynamic micropolar fluid flow and heat transfer over a vertical porous plate through a porous medium in the presence of thermal and mass diffusion with a constant heat source, Can.J.Phys., Vol.82, pp.775-790.

[18] Rahman, M.M., Sattar, M.A., Magnetohydrodynamic convective flow of a micropolar fluid past a continuously moving porous plate in the presence of heat generation/absorption, ASME.J. Heat transfer, Vol128, pp.142-153.

[19] Raptis, A., (1998), Flow of micropolar fluid past a continuously moving plate by the presence of radiation, Int. J. Heat Mass Transfer, Vol.41, pp.2865-2866.

[20] Abo-Eldahad, E.M., and Ghonaim, A.F., (2005), Radiation effect on heat transfer of a micropolar fluid through a porous medium, App. Mathematics and Computation, Vol.169, No.1, pp.500-516.

[21] Ishak, A., (2010), Thermal boundary layer flow over a stretching sheet in a micropolar fluid with radiation effect, Meccanica, Vol.45, No.3, pp.367-373.

[22] Mohammed Ibrahim, S., \& Bhaskar Reddy, N., (2013), Mass transfer and thermal radiation effects over a stretching sheet in a micropolar fluid with heat generation, International Journal of Mathematical Archive, Vol.4(1), pp.94-102.

[23] Na, T.Y., (1979), Computational Methods in Engineering Boundary Value Problems, Academic Press, New York. 


\section{AUTHORS' BIOGRAPHY}

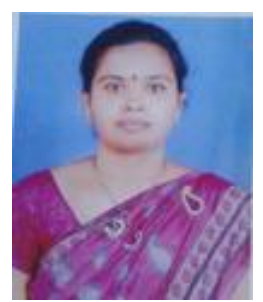

Dr. Vijaya Lakshmi S, holds a PhD in Mathematics by the University of JNTUA and is lecturer for the Department of Mathematics. Her main area of interest is the study of Fluid Dynamics. She has an experience of 11 years in academics and 1 year in Software Industry. She had published more than 5 paper $s$ in reputed International Journals.

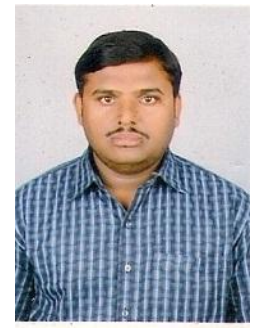

Dr. Amarnatha Reddy T, holds a PhD in Physics by the University of Acharya Nagarjuna University and is an associate professor in the Department of Physics. His main areas of interest is the study of atmospheric physics. He has an experience of 10 years in academics. He had published more than 5 paper $\mathrm{s}$ in reputed International Journals.

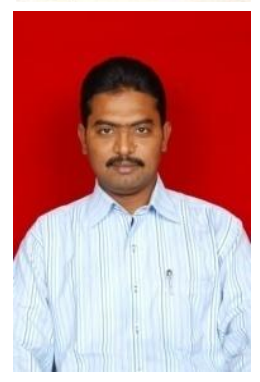

Dr. Suryanarayana Reddy M, holds a PhD in Mathematics by the University of JNUTA and is an assistant professor in the Department of Mathematics, JNTUA Pulivendua. His main areas of interest is the study of Fluid Dynamics. He has an experience of 16 years in academics. He had published more than 15 paper $\mathrm{s}$ in reputed International Journals.

Citation: S. Vijaya Lakshmi et al., " Effect of Heat Generation and Radiation on Heat Transfer in a Micropolar Fluid over a Stretching Sheet with Newtonian Heating $s$ ", International Journal of Scientific and Innovative Mathematical Research, vol. 6, no. 1, p. 21-29, 2018., http://dx.doi.org/10.20431/23473142.0601004

Copyright: () 2018 Authors. This is an open-access article distributed under the terms of the Creative Commons Attribution License, which permits unrestricted use, distribution, and reproduction in any medium, provided the original author and source are credited. 